\title{
Visualization of light beams in liquid crystal layers for demonstration of basic optical phenomena
}

S. Pasechnik, D. Shmeliova, A. Maksimochkin

S. V. Pasechnik, D. V. Shmeliova, A. G. Maksimochkin, "Visualization of light beams in liquid crystal layers for demonstration of basic optical phenomena," Proc. SPIE 9289, 12th Education and Training in Optics and Photonics Conference, 92891E (17 July 2014); doi: 10.1117/12.2070523

SDIE Event: 12th Education and Training in Optics and Photonics Conference, 2013, Porto, Portugal 


\title{
Visualization of light beams in liquid crystal layers for demonstration of basic optical phenomena.
}

\author{
Pasechnik S.V., Shmeliova D.V. and Maksimochkin A.G. \\ Moscow State University of Instrument Engineering and Computer Science, Moscow, Russia, \\ Email:s-p-a-s-m@mail.ru
}

We propose to use a liquid crystal cell as a new teaching tool for a study of basic optical phenomena like refraction and reflection of light. Such possibility is based on previously obtained experimental results [1,2] concerning propagation of light beams in the plane of a liquid crystal layer. In particular, the electrically controlled refraction and reflection of light at crossing the boundary separating regions of different orientations was registered. The scattering of light induced by thermal fluctuations of a director was used to visualize light beams. It opens new way for demonstration of optical phenomena for teaching at schools and universities.

\section{INTRODUCTION}

Liquid crystals (LC) play a key role in display industry. They also can be considered as perspective materials for non display application; in particular for fiber optics and sensor systems [3].In most cases the practical usage of LC is based on electro-optical phenomena, i.e. on possibility to change optical properties of liquid crystal media under the action of relatively weak electric fields. There are a number of electrooptical effects, like electrically induced birefringence, which can be demonstrated at study of physical backgrounds of LC applications. Nevertheless, it is possible to use such effects also for demonstration of basic optical phenomena taking place at propagation of light through optically anisotropic media, which under consideration in advanced optic courses.

In this paper we consider new possibilities connected with electrooptical effects, observed at propagation of light beams in the plane of LC layer. Such optical geometry differs from the traditional one with a normal propagation of light regarding LC layer.

Basic optical phenomena: refraction and reflection of light.

Usually, refraction and reflection of light are considered as basic phenomena observed when a light beam cross the boundary between two isotropic media with different refraction indexes. Usage of liquid crystals as optically anisotropic media makes possible to realize the alternative variant of these phenomena, when the boundary is formed inside LC layer and separates two regions with essentially different orientational structures. In our experiments [ 1,2 ] such boundary arose via application of electric field to the one part of the cell with the initially planar orientation. The schema of propagation of light in the plane of LC layer separated by the boundary into two parts is shown in Fig.1

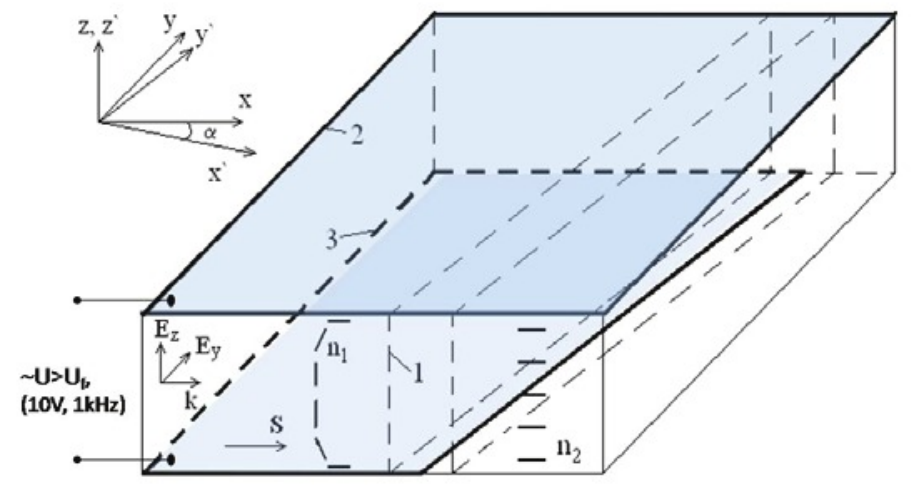

Figure 1. Schema of propagation of light through the electrically induced boundary, separating regions of homeotropic and planar orientation. 
A light beam propagates along $\mathrm{X}$-axis in the plane of LC layer confined by two glass substrates, coated with transparent electrodes. There is a gap between two electrodes on the lower substrate, oriented along y' axis, so the incident angle of a light beam is equal to $\boldsymbol{\alpha}$. The gap is needed to apply voltage $U$ to the left (1) or right (2) regions of the layer.

In the absence of applied voltage $U$ there is the initial planar orientation of a liquid crystal all over the layer provided by a proper surface treatment of the substrates and characterized by a unit vector $\mathbf{n}$ (director) which defines a local optical axis. In this case light propagates along the optical axis as an ordinary ray for any polarization state of the income wave.

Application of high enough electric voltage to the left side of the layer results in the transition from the initial planar orientation to the homeotropic one, as it is shown in Fig.1. It takes place for voltages essentially higher than the threshold voltage $U_{F}$ of Freedericksz transition, defined as:

$\mathrm{U}_{\mathrm{F}}=\pi\left[\mathrm{K}_{11} /\left(\varepsilon_{0} \Delta \varepsilon\right)\right]^{1 / 2}$,

where $\mathrm{K}_{11}$ - Frank's module, $\Delta \varepsilon$ dielectric anisotropy.

There is a big choice of LC materials with positive value $\Delta \varepsilon$ more than 10 , which results in low values of the threshold voltage $\left(\mathrm{U}_{\mathrm{F}}<1 \mathrm{~V}\right)$. So, relatively low voltages $(\mathrm{U}=5 \ldots 10 \mathrm{~V})$ are enough to suppress the orientational action of surfaces. It means that one can consider a liquid crystal in the left part of the layer as a monodomain sample with the orientation orthogonal to the initial planar orientation of the sample formed in the right part of the layer. Two parts of the layer are separated by some transition region with intermediate orientation arising due to well known fringe electric field. The computer simulation of the problem has shown that the length of this region is less than the thickness of LC layer[1] and can be approximated by a sharp boundary between two monodomain samples with different orientation of optical axes.

It is obvious that the propagation of light through the boundary depends on the polarization state of income beam which can be presented as a sum of two linear polarized waves with orthogonal directions of vector $E\left(E_{z}\right.$ and $E_{y}$ in Fig.1). For a particular case shown in Fig.1, the wave, characterized by $E_{y}$ corresponds to the ordinary ray with the same refractive index $\mathbf{n}_{\mathbf{0}}$ in two parts of the layer as vector $E_{y}$ is orthogonal everywhere to the local optical axis $\mathbf{n}$ of the sample. It means that this wave is insensitive to the electrically induced deformation of orientation and propagates through the boundary without reflection and refraction. Contrary, the wave characterized by $\mathrm{E}_{\mathrm{z}}$ propagates as an extraordinary ray with the refraction index $\mathbf{n}_{\mathbf{e}}>\mathbf{n}_{\mathbf{0}}$ in the left part of the layer and as an ordinary ray - in the right part. So, for this wave one can wait refraction and reflection of light, including the total internal reflection at crossing the boundary mentioned above.

\section{EXPERIMENTAL SETUP}

The general scheme of experiment is shown on Figure 2. We used semiconductor laser ( $640 \mathrm{~nm}$ wavelength and $10 \mathrm{~mW}$ power), optical lens (12.5 cm focus distance) to input light inside multimode fiber (20mm diameter) and polarization microscope connected with the CCD camera and PC to control LC orientation inside LC cell. Initial planar orientation in the cell obtained by using photoalignment technology [4]. In all experiments we used AC: $\mathrm{U}=5 \mathrm{~V}, \mathrm{f}=1 \mathrm{kHz}$, to do not provide electrolyze effect and destroying LC inside the cell.
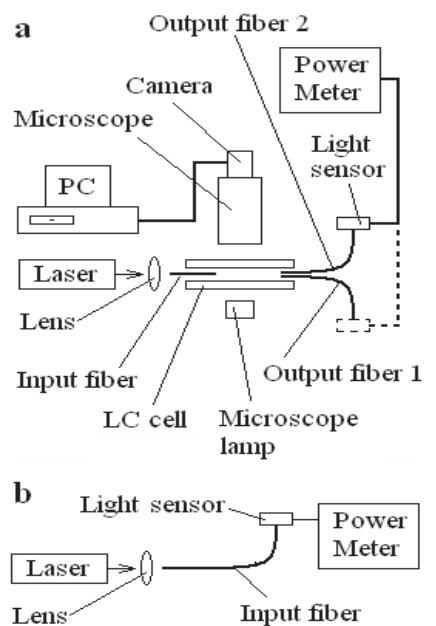

Figure 2. Experimental setup. a - general scheme, $b$ - scheme for input fiber power measurements. 


\section{REALIZATION 1}

The principle scheme of the cell used in our experiments is shown on Figure 3. Input fiber was placed between upper and lower glass directly on the ITO fascia (30 $\mu \mathrm{m}$ thickness, ITO template prepared using photolithography technology). The gap of cell was slightly larger than the diameter of multimode fiber (about $30 \mu \mathrm{m}$ ). A typical nematic LC mixture ZhK-1289 [NIOPiK production] with the principal refractive indices $\mathrm{n}_{\|}=1.68 \mathrm{n}_{\perp}=1.51$ and a high positive value of the dielectric anisotropy $\Delta \varepsilon=10.7$ was used to avoid possible electro-hydrodynamic effects and to achieve low operating voltages. The incident angle $\alpha$ needed to realize the total internal reflection is determined by the next inequality $\alpha>\sin ^{-}$ ${ }^{1}\left(\mathrm{n}_{\perp} / \mathrm{n}_{\|}\right)$. In our case $\alpha>64^{\circ}$.

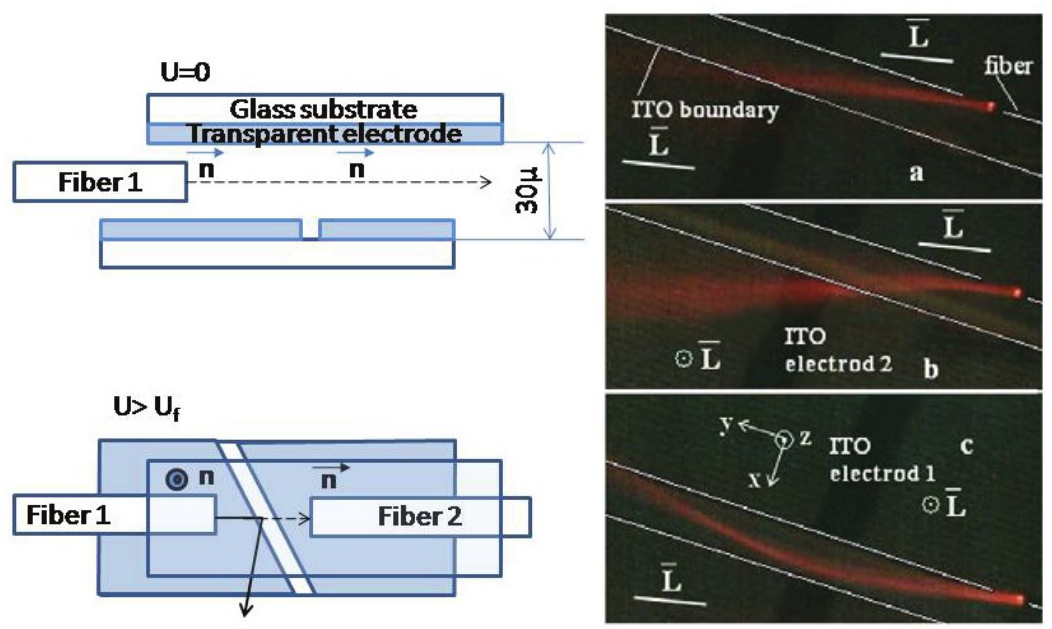

Figure 3. Schema of experimental cell and demonstration of electrically induced reflection and refraction of light beam (after [1]): (a) Initial state $(U=0)$;

(b) Refraction of light at the boundary; voltage $\mathrm{U}=2 \mathrm{~V}, 2 \mathrm{~s}$ after turning on is applied to the electrode 2;

(c) Reflection of light from the boundary; voltage $\mathrm{U}=2 \mathrm{~V}, 2 \mathrm{~s}$ after turning on is applied to the electrode 1 .

\section{REALIZATION 2}

In the second part of this article the ways of a manipulation with light beams propagating in the plane of a liquid crystal layer are described. Such geometry is useful for fiber optics applications as it is compatible with optical fibers. In particular, in our experiments we used very simple and direct way to input a light beam into LC cell by placing a glass fiber into the cell gap. Light beams propagating in the plane of a layer were easily visualized via light scattering induced by thermal fluctuations of a director. New electro-optical modes were proposed to control light beams. They are based on the primary phenomena of refraction and reflection of light on electrically induced boundary between regions of essentially different orientations [1]. The latter phenomenon can provide a waveguide mode interesting for fiber optics applications. Such mode arises due to total internal reflection at propagation of a light beam in the channel confined by the two parallel boundaries as it is shown in Figure 4. Application of electric field produces a planar-homeotropic orientational transition inside the channel which results in a waveguide regime of light propagation (Fig. $4 \mathrm{~b}$ ). In the opposite case electric field produce defocusing action when it is applied to the outer regions of the channel (Fig. 4c). The electrically controlled waveguide channels can be used in a number of fiber optics devices (shutters, attenuators, switches). 

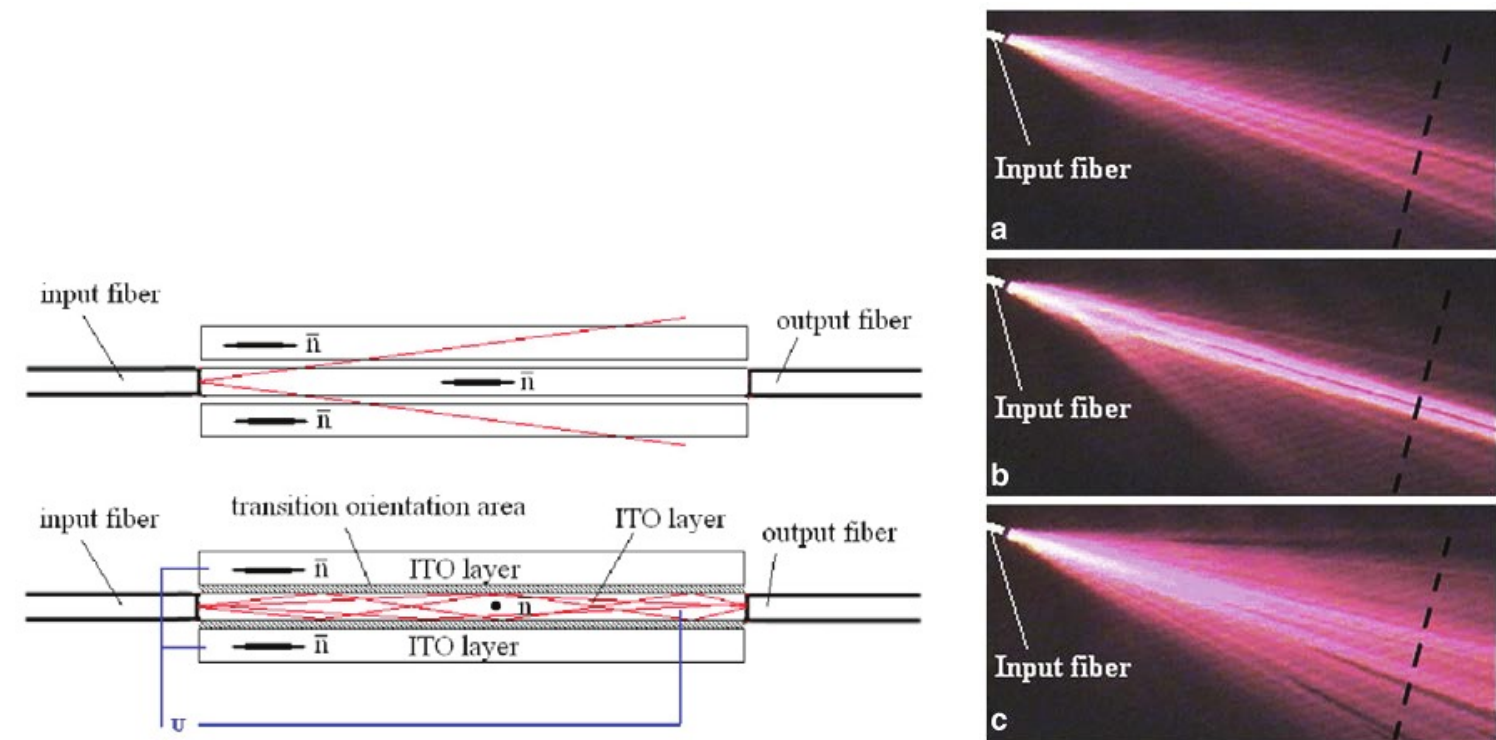

Figure 4. Experimental geometry, schema and realization of arising of electrically controlled waveguide channel (a) Light passing in homogenous planar media in the absence of electric field, (b) wave guide mode of light propagation; (c) defocusing action of electric field

\section{CONCLUSION}

The new method for a demonstration of basic optical phenomena (refraction and reflection of light, including a total internal reflection) was proposed. The method is based on observation of light beams propagating in the plane of a liquid crystal layer separated by a boundary in two regions with the essentially different orientation structures. Such configuration can be achieved by application of electric field to the one of the regions mentioned above. The advantage of the method is also connected with strong scattering of light due to thermal fluctuations of orientation which provides visualization of light beams in an anisotropic media without any additional submicron particles. We also proposed a simple way to input a light beam in a liquid crystal cell by placing a fiber directly in the gap of the cell. Such decision is attractive as it makes possible to show how the electrically controlled basic phenomena can be used in fiber optics application. In particular, a waveguide regime of light propagation controlled by electric field can be easily realized and demonstrated. In the simplest variant, a demonstration of the optical effects demands routine laboratory equipment like a microscope, a low voltage a.c. generator, a video camera and a computer. So it can be realized both in secondary schools and Universities at teaching of standard courses of optics. Such demonstrations are also useful for advanced optics teaching to illustrate some additional topics like light scattering, propagation of polarized light in anisotropic media and physical backgrounds of fiber optics applications.

\section{ACKNOWLEDGMENT}

This work was supported by Russian Ministry of Education and Science (grants 14.B37.21.0894; 14.B37.21.1914).

\section{REFERENCES}

[1] Maksimochkin, A.G. , Pasechnik, S.V., Tsvetkov, V.A. et al," Electrically controlled switching of light beams in the plane of liquid crystal layer " Optics Comm. 270 273-279 (2007).

[2] A.G. Maksimochkin, S.V. Pasechnik, G.I. Maksimochkin, V.G. Chigrinov, "Electrically controlled waveguide mode in LC layer for fiber optic applications", Optics Comm. 283 3136-3141 (2010).

[3] Pasechnik, S.V., Chigrinov, V.G. and Shmeliova, D.V. [Liquid Crystals: Viscous and Elastic Properties], Wiley-VCH, Weinheim (2009).

[4] Chigrinov, V. G., Kozenkov, V. M., and Kwok, H.-S.[Photoalignment of Liquid Crystalline Materials: Physics and Applications], Wiley, New York (2008). 